\title{
Effective treatment of esophageal carcinomas using intensity-modulated radiotherapy in combination with nedaplatin and docetaxel
}

\author{
WENJUN CHEN $^{1}$, JIANHUA WANG ${ }^{1}$, KUAILE ZHAO ${ }^{2}$, SHAOFEI YUAN ${ }^{1}$, \\ YUBIN WANG $^{1}$, YUCHENG LIN ${ }^{1}$, XUEHUI SHI ${ }^{2}$ and JIANGPING XIONG ${ }^{3}$ \\ ${ }^{1}$ Cancer Center, The Third Hospital of Wenzhou Medical College, Ruian, Zhejiang 325200; \\ ${ }^{2}$ Department of Radiotherapy, Cancer Hospital of Fudan University, Shanghai; \\ ${ }^{3}$ Department of Oncology, The First Affiliated Hospital of Nanchang University, Nanchang 330006, P.R. China
}

Received January 10, 2012; Accepted May 9, 2012

DOI: $10.3892 / \mathrm{ol} .2012 .735$

\begin{abstract}
Esophageal carcinoma is one of the most common types of cancer. Traditional surgery or radiotherapy alone is not sufficiently effective and the median survival period is nine months. The results of previous studies indicate that the efficacy of the treatment cannot be improved by increasing the doses of radiotherapy and cisplatin with 5-fluorouracil. In this study, we performed radiotherapy in combination with weekly nedaplatin plus docetaxel chemotherapy to determine the curative and toxicity effects of these treatments. The mean survival period of patients with pathological complete remission (pCR) was 47.8 months (range, 35.3-60.6), which was longer than that of the partial pathological remission (pPR) patients (mean, 17.8 months; range, 8.7-53.3). Furthermore, the relative risk of pCR mortality was lower compared with that of the non-pathological complete remission (no-pCR) patients, which were 0.088 [95\% confidence interval (CI), 0.029-0.265] and 0.08 (95\% CI, 0.028-0.225), respectively. These results suggest that the pCR patients, compared with the pPR patients (or no-pCR patients), had a significant survival advantage. The findings have shown that the chemotherapy regimen used in this study is able to significantly increase the effect of radiotherapy and reduce the toxicity effects of chemotherapy. Dose intensity-modulated radiotherapy (IMRT) reduces the
\end{abstract}

Correspondence to: Professor Wenjun Chen, Cancer Center, The Third Hospital of Wenzhou Medical College, Ruian, Zhejiang 325200, P.R. China

E-mail: wjchen2011@yahoo.cn

Dr Jiangping Xiong, Department of Oncology, The First Affiliated Hospital of Nanchang University, Yongwaizheng Road 17, Nanchang 330006, P.R. China

E-mail: jpxiong@medmail.com.cn

Key words: esophageal carcinoma, intensity-modulated radiotherapy, weekly dose of chemotherapy, nedaplatin, docetaxel irradiation volume, thus the target area is smaller than in the conventional methods.

\section{Introduction}

Esophageal carcinoma is a common cancer in China. Traditional surgery or radiotherapy alone is not sufficiently effective and the median survival period is 9 months. In 1992, Herskovic et al (1) reported a phase III clinical trial using radiotherapy (50 Gy/25 fx) and concurrent chemotherapy (cisplatin combined with 5-fluorouracil) to treat patients who had been diagnosed with local esophageal carcinomas and who had not yet undergone surgery. It was shown that the survival of patients in the radiotherapy plus chemotherapy group was markedly longer compared with that of patients in the radiotherapy group (1). al-Sarraf et al (2) and Cooper et al (3) further confirmed the advantages of the radiotherapy plus chemotherapy in prolonging survival by showing that the 3 - to 10 -year survival ratios of the radiotherapy plus chemotherapy group were $20-30 \%$. However, the 3-year survival ratio of the radiotherapy alone group was 0 . To further improve the efficacy of radiotherapy plus chemotherapy, a phase II clinical trial was performed (4) using radiotherapy plus chemotherapy with increased radiotherapy and chemotherapy doses and increased chemotherapy cycles. Although certain patients did not complete the treatments due to severe toxic effects, the results showed that no significant efficacy improvements were found. In another phase III trial (5), using the radiotherapy plus chemotherapy regimen as the control, it was found that even when the radiotherapy dose was increased to $64.8 \mathrm{~Gy}$, the local curing efficacy, median survival period and 2-year survival rate were similar to those of the control group. Certain radiotherapy plus chemotherapy regimens have been reported to be used to treat esophageal carcinomas (6-17). However, these trials show that efficacy cannot be improved by increasing the doses of radiotherapy and cisplatin with 5-fluorouracil.

In this study, we performed radiotherapy in combination with weekly nedaplatin plus docetaxel chemotherapy. The results of this study showed that our chemotherapy regimen 
is able to significantly increase the effect of radiotherapy and reduce the toxicity effects of chemotherapy. Chemotherapy with platinum has better curative effects, lower toxicity and largely increases the effect of radiotherapy. The dose intensitymodulated radiotherapy (IMRT) reduces the irradiation volume, so the target area is smaller than in the conventional method.

\section{Materials and methods}

Patients. A total of 86 patients with esophageal carcinomas were enrolled in this study and their clinical characteristics are shown in Table I. Patient consent was obtained, and the study was approved by the Medical Ethics Committee of the Third Hospital of Wenzhou Medical College (Ruian, China).

Therapeutic schedule of the experimental group. IMRT was performed. During radiotherapy, nedaplatin plus docetaxel chemotherapy was conducted once weekly using the CMS radiotherapy treatment planning system (CMS Xio 4.40). The radiation energy was $6 \mathrm{mV}$ and the radiation dose was intensity-modulated. The prescription dose was $95 \%$ of the planning target volume (PTV1) DT 60 Gy/5 times/week (2 Gy/ time). The uniformity of the clinical target volume (CTV) dose was between 95 and $105 \%$. PTV was in the 93 to $107 \%$ dose range. The chemotherapy regimens were as follows: nedaplatin $\left(25 \mathrm{mg} / \mathrm{m}^{2}\right)$ and docetaxel $\left(20 \mathrm{mg} / \mathrm{m}^{2}\right)$ were administered by intravenous drip at 1, 8, 15, 22, 29 and 36 days following radiotherapy.

Therapeutic schedule of the control group. Conventional radiotherapy was performed. During radiotherapy, 5-fluorouracil plus cisplatin chemotherapy was conducted. Radiotherapy regimens were conducted with 2 Gy administered every day for 5 consecutive days in one week. The regional radiotherapy dose was $30 \mathrm{~Gy} / 5$ times/week and the intensive radiotherapy in the shrinking field was $20 \mathrm{~Gy} / 5$ times/week. The total dose was 50 Gy/25 times/5 weeks. The dose gradient changes in the treatment tissue were $<10 \%$ and the maximum permissible dose of the spinal cord (in accordance with $2 \mathrm{~cm}$ below the beam upper bound) was $42.5 \mathrm{~Gy}$. The dose in the normal lung tissues $2 \mathrm{~cm}$ outside the gross tumor volume (GTV) did not exceed 25 Gy. The chemotherapy regimens were as follows: 5 -fluorouracil $\left(1,000 \mathrm{mg} / \mathrm{m}^{2} /\right.$ day $)$ was continuously intravenously injected with Baxter pump on the first 4 days of the 1st, 5th, 8th and 11 th weeks; cisplatin $\left(75 \mathrm{mg} / \mathrm{m}^{2}\right)$ was delivered at $1 \mathrm{mg} / \mathrm{min}$ on the first day of each course.

Therapeutic effects. The short-term effect evaluation was performed according to the RECIST solid tumor efficacy evaluation standards, including a comparison of the enhanced $\mathrm{CT}$ and the barium swallow X-ray radiography prior to and following the treatments. Patients with complete remission (CR) and partial remission (PR) were required to receive esophageal biopsy. Both imaging and pathology complete remission were classified as pathological complete remission (pCR). Partial pathological remission (pPR) indicated that imaging showed CR or PR, but the pathological examination revealed residual tumors. The effective cases that had not received histopathologic reexamination and the cases with pathological pPR were classified as non-pathological
Table I. Comparison of clinical characteristics of 86 patients with esophageal carcinomas.

\begin{tabular}{lcc}
\hline Characteristic & $\begin{array}{c}\text { EX group } \\
(\mathrm{n}=44)\end{array}$ & $\begin{array}{r}\text { CON group } \\
(\mathrm{n}=42)\end{array}$ \\
\hline $\begin{array}{l}\text { Age (years) } \\
<60\end{array}$ & $9(20)$ & $7(17)$ \\
$60-65$ & $25(57)$ & $22(52)$ \\
$66-70$ & $10(23)$ & $13(31)$ \\
Gender & & \\
Female & $5(11)$ & $4(9)$ \\
Male & $39(89)$ & $38(91)$ \\
PS & & $31(74)$ \\
$0-1$ & $29(65)$ & $11(26)$ \\
2 & $15(35)$ & \\
Weight loss in 6 months & & $11(26)$ \\
$<5 \%$ & $11(25)$ & $31(74)$ \\
$\geq 5 \%$ & $33(75)$ &
\end{tabular}

Difficulty in swallowing

No

Mild to moderate

$36(82)$

Moderate

Lesion length $(\mathrm{cm})$ $<5$

5-7

7-10

Lesion sites

Neck

Upper thoracic

Middle thoracic

21 (48)

21 (49)

Lower thoracic

4 (9)

2 (5)

UICC/02 stage

T1N0M0

1 (2)

T2-3NOMO

7 (16)

T4N0-1M0

$11(25)$

$10(24)$

T1-3N1M0

25 (57)

Type based on X-ray

$\begin{array}{lcc}\text { Medullary } & 37(83) & 35(83) \\ \text { Fungoid } & 3(7) & 3(7) \\ \text { Ulcerative } & 2(5) & 3(7) \\ \text { Other } & 2(5) & 1(3)\end{array}$

CT examination

Symmetric growth 11 (25)

Eccentric growth

$33(75)$

$30(71)$

Tumor invasion

$\begin{array}{lll}\text { Invasion } & 15(35) & 15(36) \\ \text { No } & 29(65) & 27(64)\end{array}$

No marked invasion

$29(65)$

27 (64)

EX group, experimental group; CON group, control group; PS, performance status. 
Table II. Comparison of curative effects in 86 patients with esophageal carcinomas.

\begin{tabular}{lcccc}
\hline Factor & EX group $(\mathrm{n}=44)$ & CON group $(\mathrm{n}=42)$ & $\chi^{2}$ value & P-value \\
\hline Complete remission (CR) & $47.7 \%(21 / 44)$ & $16.7 \%(7 / 42)$ & 9.44 & $<0.005$ \\
Partial pathological remission (CR+PR) & $84.1 \%(37 / 44)$ & $64.3 \%(30 / 42)$ & 2.0 & $>0.05$ \\
1-year survival rate & $75.0 \%(33 / 44)$ & $52.4 \%(22 / 42)$ & 4.77 & $<0.05$ \\
2-year survival rate & $56.8 \%(25 / 44)$ & $33.3 \%(14 / 42)$ & 4.86 & $<0.05$ \\
Median survival time (months) & $24.9(4.1-60.6)$ & $14.1(5.4-59.9)$ & 2.996 & 0.083 \\
\hline
\end{tabular}

EX group, experimental group; CON group, control group.

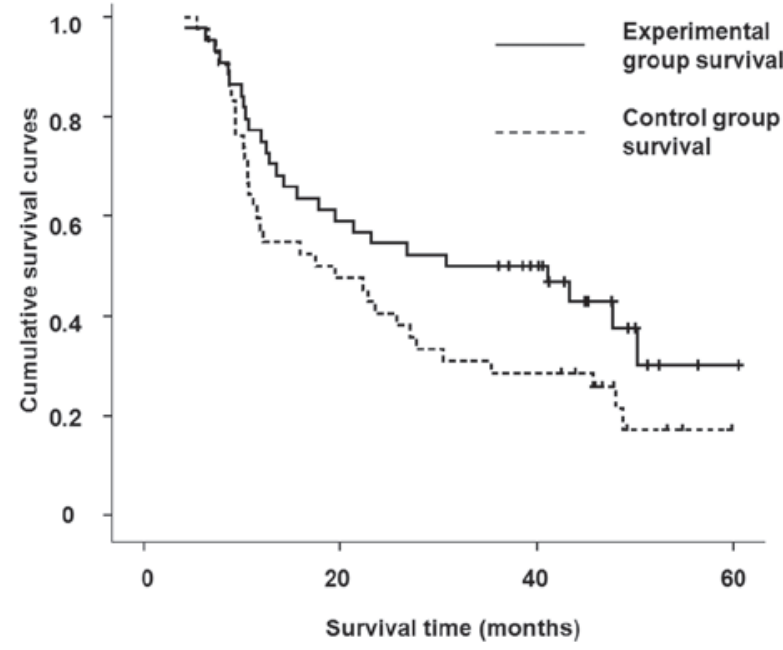

Figure 1. Cumulative survival curves of 86 esophageal cancer cases. The cumulative survival rates of patients in the experimental and control groups were calculated. The 1- and 2-year survival rates of the experimental group were higher than those of the control group $(\mathrm{P}<0.05)$.

complete remission (no-pCR). The long-term effect was evaluated through the 1 - and 2-year survival rates and survival times.

Toxicity evaluation. The toxicity of the anticancer drugs was evaluated according to the WHO acute toxicity criteria. Radiotherapy toxicity was evaluated according to the RTOG acute radiation injury grade standard.

Statistical analysis. Statistical analysis was performed using the $\chi^{2}$ test for characteristics prior to treatments in the two groups. The log-rank test was used to compare the survival curves of the two groups. The relative risk estimation of mortality was conducted using 95\% confidence intervals (95\% CIs). Survival analysis software (SPSS 13.0, SPSS, Inc., Chicago, IL, USA) was used.

\section{Results}

General data. The 86 patients who enrolled in the present study were divided into two groups, the experimental and control groups (Table I). The clinical data of these patients are listed in Table I and the clinical characteristics of the patients in the experimental and control groups were comparable.

Toxicity reaction. In the two groups, no treatment-related mortalities occurred. Grade III and IV toxicities, including radiation gastritis, weakness and radioactive esophagitis, were found in both groups. The ratio of the detected grade IV toxic reactions was $4.5 \%(2 / 44)$ in the experimental group and $21.4 \%(9 / 42)$ in the control group $\left(\chi^{2}=5.49 ; \mathrm{P}<0.05\right)$. The ratios of the detected grade III and grade IV toxicity reaction were $22.7 \%(10 / 44)$ and $42.9 \%(18 / 42)$ in the two groups, respectively $\left(\chi^{2}=3.97 ; \mathrm{P}<0.05\right)$. The ratios of life-threatening toxicities (hemorrhage, perforation, mucosal necrosis and fistula) were $4.5(2 / 44)$ and $23.5 \%(8 / 42)$ in the two groups, respectively $\left(\chi^{2}=4.40 ; \mathrm{P}<0.05\right)$. The above data show that the incidence and severity of the toxicities which occurred in the experimental group were significantly lower than those in the control group.

Efficacy evaluation. One month following chemotherapy, the 86 patients were evaluated for short-term efficacy. The survival rates and the overall survival periods are shown in Table II and the cumulative survival curves are shown in Fig. 1. The complete remission rate $(47.7$ vs. $16.7 \%$; $<<0.005)$, the 1 -year survival rate (75 vs. $52.4 \%$; $\mathrm{P}<0.05)$ and the 2 -year survival rate $(56.8$ vs. $33.3 \%$; $\mathrm{P}<0.05)$ in the experimental group were higher than those in the control group. However, a comparison of the overall survival and the median survival times in the two groups indicated that the relative mortality risk was 0.683 in the experimental group (95\% CI, 0.381-1.067). The survival rates in the two groups were not significantly different. The total efficacy (84.1 and $64.3 \%$, respectively) in the two groups was not significantly different $(\mathrm{P}>0.05)$.

Of the 86 cases, there were 69 effective cases (CR+PR), $73.9 \%(51 / 69)$ of which accepted the esophageal endoscopic biopsy pathology review. Moreover, the curative effects of $25.6 \%(22 / 86)$ of the patients were pCR and at least $33.7 \%$ $(29 / 86)$ were pPR. The survival period of the patients who did not reach clinical CR or PR was less than 24 months (range, 4.1-23.1). The efficacy comparison of the pCR and pPR or no-pCR patients (including the 18 patients who had not accepted the pathology review) in the two groups are shown in Table III and the cumulative survival curves are shown in Fig. 2. The mean survival period of the pCR patients was 47.8 months (range, 35.3-60.6), which was markedly longer than that of 
Table III. The curative effect comparison of pathological complete or partial remission in patients with esophageal cancers.

\begin{tabular}{lcccrr}
\hline Factor & $\mathrm{pCR}(\mathrm{n}=22)$ & $\mathrm{pPR}(\mathrm{n}=29)$ & No-pCR $(\mathrm{n}=64)$ & $\chi^{2}$ value & P-value \\
\hline 1-year survival rate & $100(22 / 22)$ & $72.4(21 / 29)$ & $54.7(35 / 64)$ & $7.20^{\mathrm{a}}$ & $<0.01^{\mathrm{a}}$ \\
& & & & $15.04^{\mathrm{b}}$ & $<0.005^{\mathrm{b}}$ \\
2-year survival rate & $100(22 / 22)$ & $37.9(11 / 29)$ & $29.7(19 / 64)$ & $21.10^{\mathrm{a}}$ & $<0.005^{\mathrm{a}}$ \\
& & & & $32.45^{\mathrm{b}}$ & $<0.005^{\mathrm{b}}$ \\
Median survival time (months) & $47.8(35.3-60.6)$ & $17.8(8.7-53.3)$ & $12.7(4.1-53.3)$ & $24.08^{\mathrm{a}}$ & $<0.005^{\mathrm{a}}$ \\
& & & & $31.54^{\mathrm{b}}$ & $<0.005^{\mathrm{b}}$ \\
& & &
\end{tabular}

pCR, pathological complete remission; pPR, partial pathological remission; no-pCR, non-pathological complete remission; ${ }^{\mathrm{a}} \mathrm{comparison}$ between pCR and pPR; 'bcomparison between pCR and no-pCR.

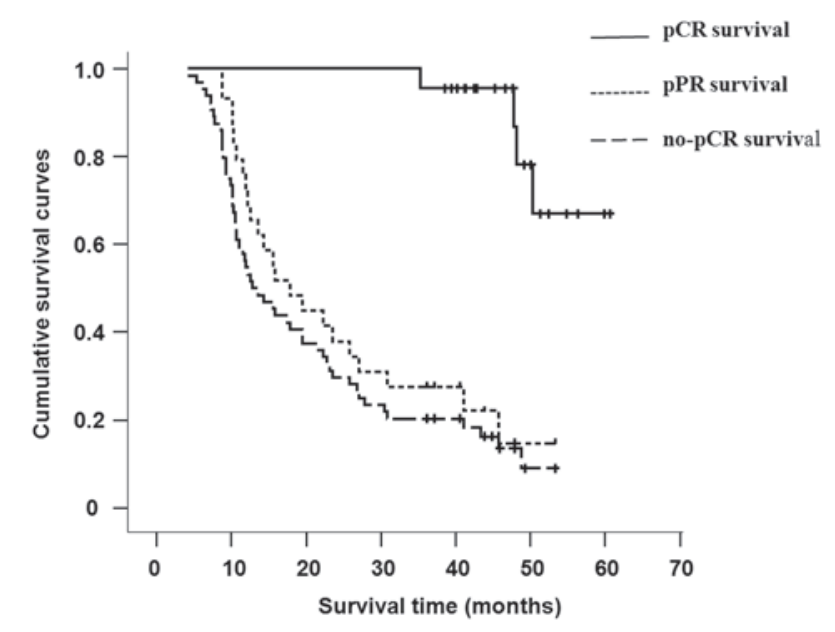

Figure 2. Cumulative survival curves of 51 esophageal cancer patients with different pathological review results. pCR, pathological complete remission; pPR, partial pathological remission; no-pCR, non-pathological complete remission.

the pPR patients (mean, 17.8 months; range, 8.7-53.3 months). Furthermore, the relative risk of pCR mortality was lower than that of the no-pCR patients, which were $0.088(95 \% \mathrm{CI}$, $0.029-0.265)$ and 0.08 (95\% CI, 0.028-0.225), respectively. The above results suggest that the pCR patients, when compared with the pPR patients (or no-pCR patients), had a significant survival advantage.

\section{Discussion}

Nedaplatin is a new platinum derivative. It has a similar efficacy to cisplatin while its toxicity to the renal and gastrointestinal tissues are low. The results of a phase II clinical trial have shown that the nedaplatin treatment of esophageal carcinoma has an efficiency of $42.9 \%$ and a low toxicity (18). Docetaxel is another drug used to treat esophageal cancers. Osaka et al (19) treated 28 cases of recurrent esophageal carcinoma with nedaplatin $\left(40 \mathrm{mg} / \mathrm{m}^{2}\right)$ and docetaxel $(30 \mathrm{mg} /$ $\mathrm{m}^{2}$ ) at intervals of 2 weeks and found that $60.7 \%$ of the cases completed the treatment. The results indicate that the CR rate was $3.6 \%$, PR rate was $35.7 \%$, the median survival time was 8.5 months and the 1-year survival rate was $15.9 \%$. Additionally, the most common toxicities were leukopenia and anemia. Non-hematological toxicities were low grade with no treatment-related mortalities. Matsumoto et al (17) used nedaplatin $\left(30 \mathrm{mg} / \mathrm{m}^{2}\right)$ and docetaxel $\left(30 \mathrm{mg} / \mathrm{m}^{2}\right)$ to treat 11 patients with relapsed or refractory esophageal carcinomas. There were no treatment-related mortalities or grade IV toxicities. Bone marrow toxicity appeared in three cases, however hematological toxicity was not observed.

Previous studies (20) have shown that the efficacy of concurrent chemoradiotherapy methods is not sufficient. Radiotherapy controls the local diseases, while chemotherapy affects the lesion outside the radiation field. Chemotherapy plays a role in radiotherapy-resistant cell populations such as hypoxia cells and enhances the radiotherapy sensitivity. Radiotherapy and chemotherapy can mutually reinforce function at the molecular level to improve survival outcome. Chemotherapy inhibits tumor proliferation following radiotherapy. No studies concerning the treatment of early esophageal carcinoma with a weekly dose of nedaplatin and docetaxel synchronous IMRT were found. Our results show that, compared with the control group, the complete remission rate and the 1- and 2-year survival rates were higher in the experimental group $(\mathrm{P}<0.05)$. Moreover, the treatment compliance in the experimental group was better $(\mathrm{P}<0.05)$ and severe and life-threatening toxicities were significantly reduced $(\mathrm{P}<0.05)$. However, the total efficiency and the median survival time in the two groups were not statistically different. As shown in the cumulative survival curve results in Fig. 1, the survival curves of the two groups are different and the survival rates of the experimental group were better than those of the control group. However, no statistically significant difference was found between the two groups, which may result from the small sample size. The 1- and 2-year survival rates and the median survival time were markedly increased in patients with complete pathology remission $(\mathrm{P}<0.05)$. Therefore, $\mathrm{pCR}$ is a key factor for obtaining better curative effects. Our findings suggest that patients who are newly diagnosed with esophageal squamous cell carcinomas that are confined in the chest may be treated with IMRT and a concurrent weekly dose of nedaplatin plus docetaxel combination chemotherapy. Furthermore, the method used in this study has improved the curative effects and lower toxicity compared with other regimens. 


\section{References}

1. Herskovic A,Martz LK, al-Sarraf M, et al: Combined chemotherapy and radiotherapy compared with radiotherapy alone in patients with cancer of the esophagus. N Engl J Med 326: 1593-1598, 1992.

2. al-Sarraf M, Martz K and Herskovic A: Progress report of combined chemoradiotherapy versus radiotherapy alone in patients with esophageal cancer: an intergroup study. J Clin Oncol 15: 277-284, 1997.

3. Cooper JS, Guo MD, Herskovic A, et al: Chemoradiotherapy of locally advanced esophageal cancer: long-term follow-up of a prospective randomized trial (RTOG 85-01). Radiation Therapy Oncology Group. JAMA 281: 1623-1627, 1999.

4. Minsky BD, Neuberg D, Kelsen DP, et al: Final report of intergroup trial 0122 (ECOG PE-289, RTOG 90-12): Phase II trial of neoadjuvant chemotherapy plus concurrent chemotherapy and high-dose radiation for squamous cell carcinoma of the esophagus. Int J Radiat Oncol Biol Phys 43: 517-523, 1999.

5. Minsky BD, Pajak TF, Ginsberg RJ, et al: INT O123 (Radiation Therapy Oncology Group 94-05) phase III trial of combinedmodality therapy for esophageal cancer: high-dose versus standard-dose radiation therapy. J Clin Oncol 20: 1167-1174, 2002

6. Tanaka Y, Yoshida K, Osada S, Yamaguchi K and Takahashi T: Docetaxel, nedaplatin, and S-1 (DGS) chemotherapy for advanced esophageal carcinoma: a phase I dose-escalation study. Anticancer Res 31: 4589-4597, 2011.

7. Matsutani T, Uchida E, Yoshida H, et al: A case of advanced esophageal carcinoma with nephrotic syndrome completely responding to chemotherapy of docetaxel, nedaplatin and 5-fluorouracil. Gan To Kagaku Ryoho 38: 439-441, 2011 (In Japanese).

8. Guo JF, Zhang B, Wu F, et al: A phase II trial of docetaxel plus nedaplatin and 5-fluorouracil in treating advanced esophageal carcinoma. Chin J Cancer 29: 321-324, 2010.

9. Tokairin Y, Kumagai Y and Yamazaki S: A case of postoperative liver metastasis of esophageal cancer remains in progression free after successfully resected. Gan To Kagaku Ryoho 36: 2462-2464, 2009 (In Japanese).

10. Noda S, Kubo N, Tanaka H, et al: A case of advanced esophageal cancer with para-aortic lymph node metastasis with complete response (CR) to combination chemotherapy including nedaplatin. Gan To Kagaku Ryoho 36: 2451-2453, 2009 (In Japanese).
11. Matsutani T, Sasajima K, Maruyama H, et al: A case of the oldest old patient with advanced esophageal cancer responding completely to the combination chemotherapy of docetaxel/5-fluorouracil/nedaplatin with radiation. Nihon Shokakibyo Gakkai Zasshi 106: 1026-1030, 2009 (In Japanese).

12. Jin J, Xu X, Wang F, et al: Second-line combination chemotherapy with docetaxel and nedaplatin for Cisplatin-pretreated refractory metastatic/recurrent esophageal squamous cell carcinoma. J Thorac Oncol 4: 1017-1021, 2009.

13. Matsutani T, Sasajima K, Kobayashi Y, et al: A case of double advanced cancer with esophageal and hypopharyngeal carcinoma responding completely to combination chemotherapy of docetaxel/5-fluorouracil and nedaplatin with radiation. Gan To Kagaku Ryoho 36: 835-838, 2009 (In Japanese).

14. Nakajima Y, Suzuki T, Haruki S, et al: A pilot trial of docetaxel and nedaplatin in cisplatin-pretreated relapsed or refractory esophageal squamous cell cancer. Hepatogastroenterology 55: $1631-1635,2008$

15. Fujita Y, Hiramatsu M, Kawai M, Sumiyoshi K, Nishimura H and Tanigawa N: Evaluation of combined docetaxel and nedaplatin chemotherapy for recurrent esophageal cancer compared with conventional chemotherapy using cisplatin and 5-fluorouracil: a retrospective study. Dis Esophagus 21: 496-501, 2008.

16. Yamazaki K, Hironaka S, Boku N, et al: A retrospective study of second-line chemotherapy for unresectable or recurrent squamous cell carcinoma of the esophagus refractory to chemotherapy with 5-fluorouracil plus platinum. Int J Clin Oncol 13: $150-155,2008$.

17. Matsumoto H, Hirai T, Hirabayashi Y, et al: A feasible study of docetaxel/nedaplatin combined chemotherapy for relapsed or refractory esophageal cancer patients as a 2nd-line chemotherapy. Gan To Kagaku Ryoho 34: 725-728, 2007 (In Japanese).

18. Taguchi T, Wakui A, Nabeya K, et al: A phase II clinical study of cis-diammine glycolato platinum, 254-S, for gastro-intestinal cancers. 254-S Gastrointestinal Cancer Study Group. Gan To Kagaku Ryoho 19: 483-488, 1992.

19. Osaka Y, Takagi Y, Hoshino S, et al: Combination chemotherapy with docetaxel and nedaplatin for recurrent esophageal cancer in an outpatient setting. Dis Esophagus 19: 473-476, 2006.

20. Kleinberg L and Forastiere A: Chemoradiation in the management of esophageal cancer. J Clin Oncol 25: 4110-4117, 2007. 